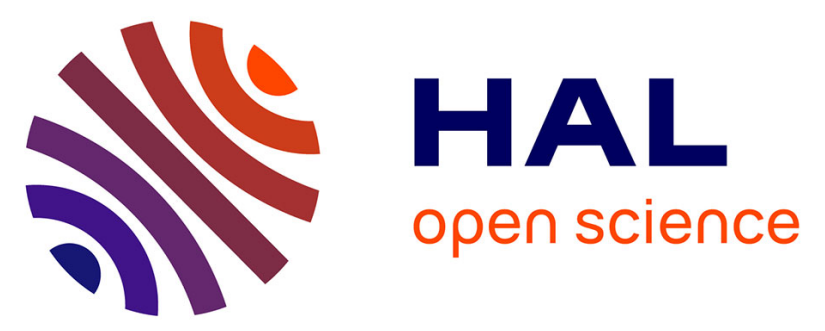

\title{
No association of the CYP3A $5 * 1$ allele with blood pressure and left ventricular mass and geometry: the KORA/MONICA Augsburg echocardiographic substudy
}

Wolfgang Lieb, Juliane Bolbrinker, Angela Döring, Hans-Werner Hense, Jeanette Erdmann, Heribert Schunkert, Reinhold Kreutz

\section{To cite this version:}

Wolfgang Lieb, Juliane Bolbrinker, Angela Döring, Hans-Werner Hense, Jeanette Erdmann, et al.. No association of the CYP3A5*1 allele with blood pressure and left ventricular mass and geometry: the KORA/MONICA Augsburg echocardiographic substudy. Clinical Science, 2006, 111 (6), pp.365-372. 10.1042/CS20060075 . hal-00479324

\section{HAL Id: hal-00479324 https://hal.science/hal-00479324}

Submitted on 30 Apr 2010

HAL is a multi-disciplinary open access archive for the deposit and dissemination of scientific research documents, whether they are published or not. The documents may come from teaching and research institutions in France or abroad, or from public or private research centers.
L'archive ouverte pluridisciplinaire HAL, est destinée au dépôt et à la diffusion de documents scientifiques de niveau recherche, publiés ou non, émanant des établissements d'enseignement et de recherche français ou étrangers, des laboratoires publics ou privés. 


\section{No association of the CYP3A5*1 allele with blood pressure and left ventricular mass and geometry: The KORA/MONICA Augsburg Echocardiographic Substudy}

Wolfgang Lieb ${ }^{1,2}$, MD; Juliane Bolbrinker ${ }^{3}$, MD; Angela Döring ${ }^{4}, \mathrm{MD}$; Hans-Werner Hense ${ }^{5}$, MD; Jeanette Erdmann ${ }^{1}$, PhD; Heribert Schunkert ${ }^{1}$, MD; Reinhold Kreutz ${ }^{3}$, MD

\footnotetext{
${ }^{1}$ Medizinische Klinik 2, Universitätsklinikum Schleswig-Holstein (UK-SH), Campus Lübeck, Lübeck, Germany

${ }^{2}$ Institut für Humangenetik, Universitätsklinikum Schleswig-Holstein (UK-SH), Campus Lübeck, Lübeck, Germany

${ }^{3}$ Department of Clinical Pharmacology, Campus Benjamin Franklin, Charité - Universitätsmedizin Berlin, Berlin, Germany,

${ }^{4}$ GSF Nationales Forschungszentrum für Umwelt und Gesundheit, Institut für Epidemiologie, Neuherberg, Germany

${ }^{5}$ Institut für Epidemiologie und Sozialmedizin, Universität Münster, Münster, Germany
}

Running head: Role of CYP3A5 for blood pressure and cardiac hypertrophy

\section{Correspondence to:}

Reinhold Kreutz, MD, FAHA

Abteilung Klinische Pharmakologie

Charité - Universitätsmedizin Berlin, Campus Benjamin Franklin

Hindenburgdamm 30,12200 Berlin, Germany,

Tel: +49-30-8445-2280, Fax: +49-30-8445-4482, e-mail: reinhold.kreutz@charite.de

Keywords: CYP3A; blood pressure; heart; hypertension; pharmacogenetics 


\section{Abstract (250 words)}

A polymorphism in the cytochrome-P450 3A (CYP3A) CYP3A5 enzyme has been implicated in blood pressure control and arterial hypertension. Carriers of the CYP $3 A 5 * 1$ allele show high while homozygous carriers of the CYP3A5*3 allele exhibit low CYP3A5 expression in the kidney, where CYP3A5 represents the major CYP3A enzyme. The aim of the current study was to investigate the association of the CYP3A5*1 allele with blood pressure, arterial hypertension, left ventricular (LV) mass and geometry in a large Caucasian population-based cohort.

We compared blood pressure, LV mass measurements, and the prevalence of hypertension between carriers $(C Y P 3 A 5 * 1 / * 1$ and $C Y P 3 A 5 * 1 / * 3$ genotypes) and noncarriers $(C Y P 3 A 5 * 3 / * 3$ genotype) of the CYP3A5*1 allele in the echocardiographic substudy of the third MONICA (MONItoring trend and determinants in CArdiovascular disease) survey.

After exclusion of 269 individuals on antihypertensive medications, 530 women and 554 men were available for analysis revealing allele frequencies of 5.8\% and $94.2 \%$ for the CYP3A5*1 and CYP3A5*3 alleles, respectively. Overall, the presence of the CYP3A5*1 allele exhibited no effect on systolic and diastolic blood pressure in both genders. One third of the individuals of this cohort were hypertensive (blood pressures $\geq 140 / 90 \mathrm{mmHg}$ ) and the genotype distribution between normotensive and hypertensive individuals revealed after adjustment for age, BMI, and gender no association between CYP3A5*1 and hypertension (OR 1.02, $\mathrm{p}=0.92$ ). Moreover, no effect of CYP3A5*1 on LV mass, wall thickness of the septal and posterior wall, and LV end-diastolic diameter was found.

CYP3A5*1 exhibits no significant effect on blood pressure, LV mass and geometry in the MONICA echocardiographic substudy. 


\section{Introduction}

Recently, a polymorphism in an isoenzyme of the cytochrome P-450 3A (CYP3A) enzyme, i.e. CYP3A5, has attracted increasing attention in cardiovascular research. This results from the fact that CYP3A5 is not only involved in drug metabolism but is also expressed in the human kidney where it is the major CYP3A enzyme [1-3]. The renal expression of CYP3A5 in epithelial cells of the proximal tubule and collecting duct [4;5] represented the basis for its implication in blood pressure and volume control and thus the pathogenesis of arterial hypertension. One potential mechanism by which CYP3A5 could mediate blood pressure effects results from the fact that CYP3A enzymes metabolize endogenous corticosteroids and mineralocorticoids in humans and animals to their 6ß-hydroxylated metabolites [3;6-8]. In the kidney, this could lead to a decreased stimulation of the mineralocorticoid receptor and thus lower sodium reabsorption via the epithelial sodium channel [6;9]. Interestingly, the expression of CYP3A5 has a strong genetic basis and is linked to a single nucleotide polymorphism (A6986G) in intron 3 of CYP3A5 that disturbs correct splicing of transcripts and leads to low expression and enzyme activity. Thus, low expression of CYP3A5 is found in homozygous carriers of the CYP3A5*3 allele while homozygous and heterozygous carriers of the CYP3A5*1 allele exhibit high expression of CYP3A5 [1;3;10;11]. Therefore, the observed higher renal expression of CYP3A5 in carriers of CYP3A5*1 could affect blood pressure regulation in these individuals.

Recent studies revealed, however, controversial results as to the role of CYP3A5* 1 for blood pressure regulation and its association with hypertension [1;12-15]. The aim of the current study therefore was to further test the role of CYP3A5*1 in a large population-based sample of Caucasian origin including both women and men in which after exclusion of individuals on antihypertensive medications - one third (33.3\%) of the individuals were hypertensive (blood pressure $\geq 140 / 90 \mathrm{~mm} \mathrm{Hg}$ ). The functional effects of CYP3A5 in the cardiovascular system could be mediated by its effects on renal sodium reabsorption $[6 ; 9 ; 16]$. Sodium intake and salt-sensitive blood pressure regulation are significantly linked to cardiac structure, $\mathrm{LVH}[17 ; 18]$ and left ventricular dysfunction [19]. Furthermore, LVH represents one of the most frequent and clinically important hypertensive organ damages [20-22] and salt-sensitive hypertension 
contributes significantly to its development [19;23]. Therefore, the effect of CYP3A5*1 on echocardiographically determined parameters of left ventricular mass and geometry was also evaluated in the present study.

\section{Methods}

\section{Study Population}

The subjects of this study participated in the echocardiographic substudy (total $n=$ 1674) of the third MONICA (MONItoring of trends and determinants in CArdiovascular disease) Augsburg survey [24], which is now continued in the framework of KORA (Cooperative Health Research in the Augsburg Area). Individuals were all Caucasians and originated from a gender- and age-stratified random sample of all German residents of the Augsburg area. The third survey represents individuals 25 to 74 years of age, and about 300 subjects for each 10-year increment. The population was studied by physical examination, blood testing, and a standardized interview including medical history, physical activity, medication and personal habits. Resting blood pressure was taken according to MONICA guidelines using the random zero sphygmomanometer after subjects had been resting in a sitting position. Individuals on antihypertensive medications were excluded and the mean of the second and third recording was calculated and employed to define hypertension as a blood pressure $\geq$ 140/90 mm Hg. Body weight in kilogram and height in meters were determined with subjects wearing light clothing. Written informed consent was obtained from all subjects, and a local ethical committee approved the study protocol.

\section{Echocardiographic Measurements}

Two-dimensional guided M-mode echocardiograms were obtained by 2 expert sonographers using the Sonos 1500 (Hewlett Packard Inc.). M-mode tracings were recorded on strip-chart paper at $50 \mathrm{~mm} / \mathrm{s}$. To reduce interobserver variability, all Mmode tracings were analyzed by a single cardiologist who was blinded for clinical and biochemical data. Measurements for M-Mode guided calculation of the left ventricular mass were taken just below the tip of the mitral valve. Only high-quality tracings that 
demonstrated optimal visualisations of endocardial and epicardial surfaces throughout the cardial cycle were used. This resulted in exclusion of $16 \%$ of potential subjects. Left ventricular end-diastolic diameter (LVEDD), septal wall (SWT) and posterior wall (PWT) thickness were measured according to the recommendations of the American Society of Echocardiography (ASE) [25]. Left ventricular mass (LVM) was calculated using the formula LVM $(\mathrm{g})=0.8 \times 1.04\left[(\mathrm{LVEDD}+\mathrm{SWT}+\mathrm{PWT})^{3}-\mathrm{LVEDD}^{3}\right]+0.6$ as described by Devereux et al. [26]. LVM was indexed to body surface area (BSA), $\mathrm{LVMI}=\mathrm{LVM} / \mathrm{BSA}$. Left ventricular hypertrophy was defined as LVMI $>134 \mathrm{~g} / \mathrm{m}^{2}$ in men and $\mathrm{LVMI}>110 \mathrm{~g} / \mathrm{m}^{2}$ in women [27].

\section{Biochemical Analyses}

Blood was drawn for biochemical analyses from non-fasting subjects. Creatinine was assessed quantitatively with an enzymatic colorimetric test (Hitachi 717, Boehringer Mannheim, Germany). Glomerular filtration rate was estimated (eGFR) using the modified MDRD (Modification of Diet in Renal Disease) equation: $\operatorname{eGFR}\left(\mathrm{ml} / \mathrm{min} / 1.73 \mathrm{~m}^{2}\right)=186 \times(\mathrm{Scr})^{-1.154} \times(\text { Age })^{-0.203} \times(0.742$ if female $)[28]$.

\section{CYP3A5 genotype determination}

Genotyping was performed by real-time PCR as reported [29]. The ABI PRISM ${ }^{\circledR} 7000$ SDS instrument in conjunction with the ABI TaqMan Universal Master Mix (Applied Biosystems, Darmstadt, Germany) was used to perform the assays [30]. Appropriate primers and fluorogenic probes were designed with the Primer Express ${ }^{\circledR}$ software. Fluorogenic probes were synthesized by TIB Molbiol (Berlin, Germany) and primers were obtained from Proligo (Paris, France). Primers were selected according to the sequence available at GenBank accession number NG_000004 [31]. The following probes and primers were used for genetic determination of the CYP3A*1 and CYP3A5*3 alleles (nt 260167G $>$ A): the fluorogenic probes were 5'VICTGTCTTTCAgTATCTCTT (18-mer, nt 260158-260175) and 5'FAMTGTCTTTCAaTATCTCTTC (19-mer, nt 260158-260176), and the primers were ACCCAGCTTAACGAATGCTCTACT (forward, 24-mer, nt 260098-260121) and GAAGGGTAATGTGGTCCAAACAG (reverse, 23-mer, nt 260178-260200). The TaqMan assays were performed in 384-well plates prepared with the GENESIS Freedom pipetting robot from TECAN (Crailsheim, Germany). For the present study, 
1359 individuals were successfully genotyped.

\section{Statistical Analyses}

To determine whether the genotypes deviated from Hardy-Weinberg equilibrium, actual and predicted genotype counts were compared by a $\chi^{2}$ goodness-of-fit test with 1 degree of freedom. Least square means for systolic (SBP) and diastolic (DBP) blood pressure as well as for pulse pressure (PP, defined as SBP-DBP) adjusted for age, body mass index (BMI), and gender were calculated for all genotype groups. Due to the expected small number of individuals homozygous for the CYP3A5*1 allele and according to the expectation that CYP3A5*1 allele carriers have a higher CYP3A5 enzyme activity $[1 ; 10 ; 11]$, CYP3A5*1/*1 individuals and CYP3A5*1/*3 individuals were analyzed as a combined group against the CYP3A5*3/*3 homozygotes with low expression [29]. Thus, the influence of presence or absence of the CYP3A5*1 allele in a dominant model was primarily analyzed and descriptive p-values of a two-tailed t-test for independent groups are reported. All analyses were performed for the entire population and stratified by gender. For description, a logistic regression model with hypertension (presence vs. absence of hypertension) as dependent variable and genotype, age, gender, and BMI as explanatory variables was developed. Odds ratios (OR), 95\% confidence intervals (CI) and two-tailed descriptive $p$-values of the WALD- $\chi^{2}$-test are presented for the entire population and for males and females separately. Echocardiographical data and eGFR measurements according to CYP3A5 genotypes were descriptively compared using multiple linear regression. Echocardiographical data were adjusted for age, BMI, SBP, and gender. Furthermore, eGFR according to CYP3A5 were considered by calculating least square means adjusted for age, gender, and diabetes. In addition, adjusted mean differences between carriers and non-carriers of the CYP3A5*1 allele with corresponding 95\% confidence intervals (CI) were calculated for SBP, DBP, LVMI, and eGFR. All calculations were performed using SPSS version 12.1 (SPSS, Chicago). All p-values should be considered descriptive.

\section{Results}

In total, 670 women and 689 men were successfully genotyped. 6 individuals were excluded because of missing covariates. Furthermore, 269 individuals with 
antihypertensive medication (131 men, 138 women) were excluded, leaving 530 women and 554 men for the final analyses. The allele frequencies of the CYP3A5*1 and CYP3A5*3 allele were $5.8 \%$ and $94.2 \%$, respectively.

The CYP3A5*1/*1, CYP3A5*1/*3, and CYP3A5*3/*3 genotypes were found in $0.5 \%$, $10.6 \%$, and $88.9 \%$ of the population. These frequencies do not deviate from those predicted by Hardy-Weinberg equilibrium and were similar to those reported in Caucasians [9].

Table 1 displays baseline characteristics, blood pressure levels, left ventricular mass measurements as well as eGFR measurements according to CYP3A5 genotype in the overall study population and stratified by gender. Carriers of the CYP3A5*1 allele were slightly younger than non-carriers, while no differences in BMI and the prevalence of diabetes were found in men and women between carriers and non-carriers of the CYP3A5*1 allele. The baseline characteristics of the study population, stratified by hypertension status and gender are shown in Table 2.

\section{Association of CYP3A5*1 with blood pressure measurements and hypertension}

In the overall analysis the presence of the CYP3A5*1 allele exhibited no effect on systolic and diastolic blood pressure and pulse pressure in both men and women (Table 1). The observed adjusted difference between CYP3A5*1/*1 + CYP $3 A 5 * 3 / * 1$ individuals compared to CYP3A5*3/*3 individuals is $-0.40(95 \% \mathrm{CI}-3.45$ to +2.65$)$ $\mathrm{mm} \mathrm{Hg}$ for systolic blood pressure and $0.31(95 \% \mathrm{CI}-1.67$ to +2.30$) \mathrm{mm} \mathrm{Hg}$ for diastolic blood pressure. Likewise, no association of CYP3A5*1 allele with the prevalence of hypertension was found in our cohort in multivariate models adjusting for age, BMI, and gender (OR 1.02, $\mathrm{p}=0.92$; Table 3). Overall, 361 (33.3\%) individuals were diagnosed with hypertension (Table 3). In normotensive females a somewhat higher allele frequency for $C Y P 3 A 5^{*} 1$ was observed compared to the other groups. However, no association between the presence of CYP3A5*1 and hypertension was detected in separate analysis in men and women (Table 3).

\section{Association of CYP3A5*1 with left ventricular mass and geometry}

Left ventricular mass measurements could be determined echocardiographically in 942 individuals (464 men and 478 women). No association of the CYP3A5*1 allele with LVMI, SWT, PWT and LVEDD could be found in multivariate analyses in males and 
females and in the combined analysis of both genders (Table 1). For LVMI, the observed adjusted difference between CYP3A5*1/*1+CYP3A5*3/*1 individuals and CYP3A5*3/*3 individuals is $-0.73(95 \%$ CI -3.81 to +2.34$) \mathrm{g} / \mathrm{m}^{2}$. The CYP3A5 genotype distribution was slithly different between individuals with $(* 3 / * 3: 93.8 \%$, *3/*1: $3.1 \% ; * 1 / * 1: 3.1 \%)$ and without LVH $(* 3 / * 3: 88.4 \% ; * 3 / * 1: 11.3 \% ; * 1 / * 1$ : $0.3 \% ; \mathrm{p}=0.022)$. However, in multivariate analyses, adjusting for age, BMI, SBP, and gender, no relevant $\mathrm{LVH}$ protecting effect associated with the CYP3A5*1 allele could be observed (OR: 0.66 [95\% CI 0.15-2.95]; $\mathrm{p}=0.59$ ).

\section{Association of CYP3A5*1 with eGFR}

We also analyzed the association of $C Y P 3 A 5^{*} 1$ with renal function represented by estimated GFR (eGFR) values, which demonstrated no association in the entire popuation and in women (Table 1). In the entire population, the observed adjusted difference assuming a dominant model for the rare CYP3A5*1 allele is 1.06 (95\% CI -3.02 to +5.13$)$ In men, a weak evidence for association was found $(p=0.045)$. The genotype distribution was not different between individuals with mildly impaired renal function (eGFR $<90 \mathrm{ml} / \mathrm{min} / 1.73 \mathrm{~m}^{2}$ ) and individuals with normal renal function (eGFR $\geq 90 \mathrm{ml} / \mathrm{min} / 1.73 \mathrm{~m}^{2}$ ), neither in the combined analysis of both genders nor in separate analysis according to gender (data not shown).

\section{Discussion}

The research on the role of the CYP3A5 polymorphism in blood pressure regulation has been stimulated by the initial study in a small group of African-Americans in which higher blood pressures were reported in individuals carrying the CYP3A5*1 allele [1]. Subsequently, additional studies analyzed the role of CYP3A5 on blood pressure in different populations producing controversial results [9;12-14;29]. In this regard it is important to consider that the frequency of carriers of $C Y P 3 A 5 * 1$, i.e. high expressors of CYP3A5, shows extreme variations across human populations [3] and is significantly correlated with the distance from the equator with the highest frequency of approximately $70 \%$ observed in Africans and the lowest with approximately $10 \%$ found in European Caucasians [16]. Consequently, the relevance of CYP3A5*1 may vary 
between different ethnic populations [3]. Due to the relative low frequency in Caucasians it appears particularly important to study large cohorts in this ethnic group. In the study by Ho et al. no association between CYP3A ${ }^{*} 1$ and hypertension was found in white patients from North America, while the proportion of salt-sensitive subjects was higher in white hypertensive $C Y P 3 A 5 * 1$ carriers compared to non-carriers [12]. In a population of elderly individuals (age $\geq 75$ years) from Finland it was shown, that the proportion of individuals carrying the $C Y P 3 A 5^{*} 1$ allele was significantly higher in hypertensives compared to normotensive elderly subjects suggesting a role of CYP3A5*1 for hypertension development in elderly Caucasian subjects [13]. More recently, Bochud et al. analyzed the effect of CYP3A5 polymorphisms on ambulatory blood pressure and GFR in a cohort of 375 individuals recruited from family with at least two hypertensive siblings [15]. The authors obtained an allele frequency of $43.7 \%$ for CYP3A5*1 compared to $5.8 \%$ in the Caucasian cohort of the current report. Although crude blood pressure levels were similar between carriers and non-carriers of the CYP3A5*1 allele in the study by Bochud et al., the authors detected a significant positive interaction between $C Y P 3 A 5 * 1$ and age for systolic and diastolic ambulatory blood pressure during daytime but not at night. They reported that CYP $3 A 5^{*} 1$ carriers demonstrated an age-related significant blood pressure increase. Interestingly, Bochud et al. also investigated the effect of CYP $3 A 5 * 1$ on renal sodium handling by fractional excretion of lithium and found that non-carriers of $C Y P 3 A 5^{*} 1$ decreased their proximal tubular sodium reabsorption with age, while CYP3A5*1 carriers did not. Further analysis between urinary sodium excretion - reflecting dietary salt intake - and ambulatory blood pressure indicated, that $C Y P 3 A 5 * 1$ carriers tended to increase their night-time blood pressure with increasing dietary salt-intake more than non-carriers. The authors suggested [15] that carriers of CYP3A5*1 appear to be more salt-sensitive than non-carriers and supported a role for CYP3A5*1 in salt-sensitive hypertension, which might be particularly important with increasing age [16;32].

In contrast, $C Y P 3 A 5 * 1$ was found to be associated with lower systolic blood pressure, but not diastolic blood pressure, in a homogenous group of 115 young white, male students with normal or mildly elevated blood pressures [14]. This study was in agreement with our recent investigation in a large population-based Caucasian cohort, i.e. the Prevention of REnal and Vascular ENd stage Disease (PREVEND) study 
population, in which we also determined lower systolic blood pressures and pulse pressures but not diastolic blood pressures in carriers of the CYP3A5*1 allele [9]. Taken together the latter two studies are in agreement with the postulated mechanism that renal CYP3A5 expression confers a blood pressure lowering effect by decreasing renal sodium reabsorption [9]. Nevertheless, these findings are at variance with the data obtained in the current report, in which we found no association between CYP3A5*1 and any blood pressure phenotype in both men and women. This is of interest since the subgroup analysis in the PREVEND study population according to gender revealed that CYP3A5*1 exhibited a significant effect on systolic blood pressure and pulse pressure only in females but not in males [9].

Importantly, in the current analysis of the KORA/MONICA echocardiographic substudy population we could not find an association of the CYP3A5*1allele with left ventricular mass measurements or $\mathrm{LVH}$ in multivariate models. The finding on left ventricular echocardiographic parameters is in line with the observation reported by Fromm et al. who also performed echocardiographic analyses of left ventricular mass in their young male individuals and detected no effect of $C Y P 3 A 5^{*} 1$ on cardiac parameters although they detected significant lower systolic blood pressures in carriers of CYP3A5*1 [14].

In addition to blood pressure effects Givens et al. reported in their small study in young healthy Afro-Americans an association between CYP3A5*1 and GFR measurements, i.e. creatinine clearance [1]; this observation was only made in females but not in males by comparing 10 female CYP3A5*1 carriers with 5 female non-carriers. This finding should be viewed with caution, since the numbers in the latter study were clearly very small and a confounding factor due to differences in body-mass-index in females could not be excluded in this study because values for creatinine clearance were not adjusted to body surface area [1]. In the more recent study by Bochud et al. CYP3A5*1 exhibited a significant effect on GFR measured by inulin clearance $(\mathrm{P}=0.045)$, however, the significance disappeared after the adjustment for body surface $(\mathrm{P}=0.093)$, while no significant association with creatinine clearance was observed [15]. In contrast, Ho et al. found in their study no effect of CYP $3 A 5^{*} 1$ on creatinine clearance and urinary albumin excretion in both Afro-Americans and Caucasian individuals. Similarly, Fromm et al. 
found also no effect of CYP3A5*1 on GFR measurements in their carefully studied cohort of young Caucasian individuals [14]. In this regard a limitation of our study results from fact that renal sodium handling was not studied and direct GFR measurements were not available. Nevertheless, determination of eGFR values by the MDRD equation [28] revealed overall no significant effect of CYP3A5*1, while only a subgroup analysis according to gender indicated higher GFR levels in male but not female carriers of CYP3A5*1. Taken together, as evident from the controversial data reported so far a consistent effect of CYP3A5*1 on GFR is far from being established.

Although the number of 1084 individuals analyzed herein in the KORA/MONICA study population is still considerable high and exceeds by far the figures reported in Caucasians by others $[1 ; 13 ; 14]$ it is still significantly lower compared to 6777 individuals analyzed in the PREVEND study [9]. Therefore, the negative result in the current study may be in part related to a lack of statistical power. The observed adjusted difference between CYP3A5*1/*1 + CYP3A5*3/*1 individuals compared to CYP3A5*3/*3 individuals is $-0.40(95 \% \mathrm{CI}-3.45$ to +2.65$) \mathrm{mm} \mathrm{Hg}$ for SBP and 0.31 $(95 \%$ CI -1.67 to +2.30$) \mathrm{mm} \mathrm{Hg}$ for DBP. Thus, with a confidence of $95 \%$, the true difference between the groups lies within this interval and a mean difference smaller than -3.45 or greater than $2.65 \mathrm{~mm} \mathrm{Hg}$ for SBP and smaller than -1.67 or greater than $2.30 \mathrm{~mm} \mathrm{Hg}$ for DBP can be excluded. For LVMI, differences smaller than -3.81 or greater than $2.34 \mathrm{~g} / \mathrm{m}^{2}$ between carriers and non-carriers of the CYP3A5*1 allele can be excluded with $95 \%$ confidence.

Another important issue relates to the higher frequency of individuals with hypertension (33.3\%; defined as BP $\geq 140 / 90 \mathrm{~mm} \mathrm{Hg}$ ) analyzed in the current KORA/MONICA study compared to PREVEND in which hypertension was less prevalent (22\%). In this regard it appears possible that the functional effect of CYP3A5*1 depends on the blood pressure level and stage of hypertension and might be more relevant - in agreement with our recent analysis in PREVEND [9] and the study by Fromm et al. [14] - at lower blood pressure values. The biological effect at higher blood pressure levels could be blunted or eliminated due to other genetic factors that come into play and/or compensatory mechanisms. The potential relevance of adaptive mechanisms has been highlighted in the recent study in young male individuals with normal or mildly 
elevated blood pressures [14]. In this study the authors could demonstrate that 96 individuals with the CYP3A5*3/*3 genotype demonstrated on average $5 \mathrm{mmHg}$ higher systolic blood pressure values compared to the 9 carriers of the CYP3A5*1 allele, while serum aldosterone levels were significantly lower in CYP3A5*3/*3 suggesting a compensatory suppression of aldosterone in response to higher systolic blood pressures in these individuals [14]. Consequently, it appears of interest to further pursue this hypothesis and to perform both experimental and clinical studies on the role of CYP3A5 in blood pressure control.

Acknowledgements: This study was supported by a grant from the Bundesministerium für Bildung und Forschung (Dr. Kreutz), Nationales Genomforschungsnetz (Grant KGCV1, 01GS0416 to Dr. Kreutz; 01GS0418 to Drs Schunkert and Erdmann). 


\section{References:}

1. Givens,R.C., Lin,Y.S., Dowling,A.L. et al. (2003) CYP3A5 genotype predicts renal CYP3A activity and blood pressure in healthy adults. J Appl.Physiol 95, 1297-1300.

2. Haehner,B.D., Gorski,J.C., Vandenbranden,M. et al. (1996) Bimodal distribution of renal cytochrome P450 3A activity in humans. Mol.Pharmacol. 50, 52-59.

3. Wojnowski,L. (2004) Genetics of the variable expression of CYP3A in humans. Ther Drug Monit. 26, 192-199.

4. Schuetz,E.G., Schuetz,J.D., Grogan,W.M. et al. (1992) Expression of cytochrome P450 3A in amphibian, rat, and human kidney. Arch.Biochem.Biophys. 294, 206214.

5. Murray,G.I., McFadyen,M.C., Mitchell,R.T., Cheung,Y.L., Kerr,A.C. and Melvin,W.T. (1999) Cytochrome P450 CYP3A in human renal cell cancer. Br.J Cancer 79, 1836-1842.

6. Morris,D.J., Latif,S.A., Rokaw,M.D., Watlington,C.O. and Johnson,J.P. (1998) A second enzyme protecting mineralocorticoid receptors from glucocorticoid occupancy. Am J Physiol 274, C1245-C1252.

7. Wrighton,S.A., Brian,W.R., Sari,M.A. et al. (1990) Studies on the expression and metabolic capabilities of human liver cytochrome P450IIIA5 (HLp3). Mol Pharmacol 38, 207-213.

8. Watlington,C.O., Kramer,L.B., Schuetz,E.G. et al. (1992) Corticosterone 6 betahydroxylation correlates with blood pressure in spontaneously hypertensive rats. Am J Physiol 262, F927-F931.

9. Kreutz,R., Zuurman,M., Kain,S., Bolbrinker,J., de Jong,P.E. and Navis,G. (2005) The role of the cytochrome P450 3A5 enzyme for blood pressure regulation in the general Caucasian population. Pharmacogenet.Genomics 15, 831-837.

10. Kuehl,P., Zhang,J., Lin,Y. et al. (2001) Sequence diversity in CYP3A promoters and characterization of the genetic basis of polymorphic CYP3A5 expression. Nat.Genet. 27, 383-391.

11. Lin,Y.S., Dowling,A.L., Quigley,S.D. et al. (2002) Co-regulation of CYP3A4 and CYP3A5 and contribution to hepatic and intestinal midazolam metabolism. Mol.Pharmacol. 62, 162-172.

12. Ho,H., Pinto,A., Hall,S.D. et al. (2005) Association between the CYP3A5 genotype and blood pressure. Hypertension 45, 294-298.

13. Kivisto,K.T., Niemi,M., Schaeffeler,E. et al. (2005) CYP3A5 Genotype is Associated with Diagnosis of Hypertension in Elderly Patients: Data from the 
DEBATE Study. Am J Pharmacogenomics. 5, 191-195.

14. Fromm,M.F., Schmidt,B.M., Pahl,A., Jacobi,J. and Schmieder,R.E. (2005) CYP3A5 genotype is associated with elevated blood pressure. Pharmacogenet.Genomics 15, 737-741.

15. Bochud,M., Eap,C.B., Elston,R.C. et al. (2006) Association of CYP3A5 genotypes with blood pressure and renal function in African families. J.Hypertens. 24, 923929.

16. Thompson,E.E., Kuttab-Boulos,H., Witonsky,D., Yang,L., Roe,B.A. and Di,R.A. (2004) CYP3A variation and the evolution of salt-sensitivity variants. Am J Hum Genet 75, 1059-1069.

17. Schmieder,R.E., Messerli,F.H., Garavaglia,G.E. and Nunez,B.D. (1988) Dietary salt intake. A determinant of cardiac involvement in essential hypertension. Circulation 78, 951-956.

18. Langenfeld,M.R. and Schmieder,R.E. (1995) Salt and left ventricular hypertrophy: what are the links? J.Hum.Hypertens. 9, 909-916.

19. Rothermund,L., Vetter,R., Dieterich,M. et al. (2002) Endothelin-A receptor blockade prevents left ventricular hypertrophy and dysfunction in salt-sensitive experimental hypertension. Circulation 106, 2305-2308.

20. Koren,M.J., Mensah,G.A., Blake,J., Laragh,J.H. and Devereux,R.B. (1993) Comparison of left ventricular mass and geometry in black and white patients with essential hypertension. Am J Hypertens 6, 815-823.

21. Kannel,W.B. (2000) Risk stratification in hypertension: new insights from the Framingham Study. Am J Hypertens 13, 3S-10S.

22. Lorell,B.H. and Carabello,B.A. (2000) Left ventricular hypertrophy: pathogenesis, detection, and prognosis. Circulation 102, 470-479.

23. du Cailar,G., Ribstein,J. and Mimran,A. (2002) Dietary sodium and target organ damage in essential hypertension. Am.J.Hypertens. 15, 222-229.

24. Schunkert,H., Hengstenberg,C., Holmer,S.R. et al. (1999) Lack of association between a polymorphism of the aldosterone synthase gene and left ventricular structure. Circulation 99, 2255-2260.

25. Devereux,R.B., Lutas,E.M., Casale,P.N. et al. (1984) Standardization of M-mode echocardiographic left ventricular anatomic measurements. J Am Coll.Cardiol. 4, 1222-1230.

26. Devereux,R.B., Alonso,D.R., Lutas,E.M. et al. (1986) Echocardiographic assessment of left ventricular hypertrophy: comparison to necropsy findings. Am J Cardiol. 57, 450-458. 
27. Schunkert,H., Hense,H.W., Muscholl,M. et al. (1997) Associations between circulating components of the renin-angiotensin-aldosterone system and left ventricular mass. Heart 77, 24-31.

28. Levey,A.S., Coresh,J., Balk,E. et al. (2003) National Kidney Foundation practice guidelines for chronic kidney disease: evaluation, classification, and stratification. Ann.Intern.Med. 139, 137-147.

29. Kreutz,R., Zurcher,H., Kain,S., Martus,P., Offermann,G. and Beige,J. (2004) The effect of variable CYP3A5 expression on cyclosporine dosing, blood pressure and long-term graft survival in renal transplant patients. Pharmacogenetics 14, 665671.

30. Ranade,K., Chang,M.S., Ting,C.T. et al. (2001) High-throughput genotyping with single nucleotide polymorphisms. Genome Res. 11, 1262-1268.

31. Smith,G., Stubbins,M.J., Harries,L.W. and Wolf,C.R. (1998) Molecular genetics of the human cytochrome P450 monooxygenase superfamily. Xenobiotica 28, $1129-1165$.

32. Weinberger,M.H. (1996) Salt sensitivity of blood pressure in humans. Hypertension 27, 481-490. 
Table 1. Baseline characteristics, blood pressure and left ventricular mass measurements, as well as eGFR measurements according to the CYP3A5 genotype in the entire study population and stratified by gender.

\begin{tabular}{|c|c|c|c|c|c|c|c|c|c|c|c|c|}
\hline & \multicolumn{3}{|c|}{ Combined } & \multirow[b]{2}{*}{$\mathbf{p}$} & \multicolumn{3}{|c|}{ Men } & \multicolumn{5}{|c|}{ Women } \\
\hline & $* 3 / * 3$ & $* 3 / * 1$ & $* 1 / * 1$ & & $* 3 / * 3$ & $* 3 / * 1$ & $* 1 / * 1$ & p & $* 3 / * 3$ & $* 3 / * 1$ & $* 1 / * 1$ & p \\
\hline \multicolumn{13}{|l|}{ Baseline Characteristics } \\
\hline $\mathbf{N}$ & 964 & 115 & 5 & & 501 & 52 & 1 & & 463 & 63 & 4 & \\
\hline Age, years $($ mean $\pm \mathrm{SD})$ & $49.2 \pm 13.3$ & $45.5 \pm 13.0$ & $50.6 \pm 15.6$ & 0.007 & $49.5 \pm 13.6$ & $45.3 \pm 14.5$ & 64.0 & 0.056 & $48.9 \pm 13.1$ & $45.7 \pm 11.7$ & $47.3 \pm 15.8$ & 0.066 \\
\hline BMI, $\mathrm{kg} / \mathrm{m}^{2}$ (means $\left.\pm \mathrm{SD}\right)$ & $26.2 \pm 3.8$ & $26.1 \pm 3.9$ & $26.9 \pm 2.2$ & 0.79 & $26.7 \pm 3.3$ & $26.6 \pm 3.6$ & 24.1 & 0.75 & $25.7 \pm 4.3$ & $25.7 \pm 4.2$ & $27.7 \pm 1.8$ & 0.87 \\
\hline Diabetes, n (\%) & $25(2.6)$ & $1(0.9)$ & 0 & 0.35 & $16(3.2)$ & $1(1.9)$ & 0 & 0.99 & $9(1.9)$ & 0 & 0 & 0.61 \\
\hline \multicolumn{13}{|c|}{ Blood pressure measurements ${ }^{\mathrm{a}}$} \\
\hline $\mathbf{N}$ & 964 & 115 & 5 & & 501 & 52 & 1 & & 463 & 63 & 4 & \\
\hline SBP, $\mathrm{mm} \mathrm{Hg}$ & $131.3 \pm 0.5$ & $130.8 \pm 1.5$ & $135.0 \pm 7.2$ & 0.80 & $134.8 \pm 0.7$ & $134.2 \pm 2.2$ & $161.1 \pm 15.8$ & 0.99 & $127.7 \pm 0.7$ & $127.2 \pm 2.0$ & $126.6 \pm 8.0$ & 0.78 \\
\hline DBP, $\mathrm{mm} \mathrm{Hg}$ & $80.1 \pm 0.3$ & $80.3 \pm 1.0$ & $82.3 \pm 4.7$ & 0.76 & $82.4 \pm 0.5$ & $81.6 \pm 1.5$ & $102.3 \pm 10.5$ & 0.83 & $77.7 \pm 0.5$ & $78.8 \pm 1.3$ & $76.0 \pm 5.1$ & 0.51 \\
\hline pulse pressure, $\mathrm{mm} \mathrm{Hg}$ & $51.2 \pm 0.4$ & $50.4 \pm 1.2$ & $52.7 \pm 5.5$ & 0.55 & $52.4 \pm 0.6$ & $52.6 \pm 1.7$ & $58.8 \pm 12.5$ & 0.87 & $50.0 \pm 0.6$ & $48.4 \pm 1.5$ & $50.5 \pm 6.1$ & 0.35 \\
\hline \multicolumn{13}{|l|}{ LV mass measurements ${ }^{b}$} \\
\hline $\mathbf{N}$ & 834 & 104 & 4 & & 418 & 46 & 0 & & 416 & 58 & 4 & \\
\hline LVMI, $g / \mathrm{m}^{2}$ & $85.0 \pm 0.5$ & $84.0 \pm 1.5$ & $91.4 \pm 7.6$ & 0.64 & $92.1 \pm 0.8$ & $92.3 \pm 2.5$ & & 0.95 & $78.0 \pm 0.7$ & $76.0 \pm 1.8$ & $84.2 \pm 6.7$ & 0.40 \\
\hline Septal wall, mm & $10.2 \pm 0.1$ & $10.1 \pm 0.1$ & $11.1 \pm 0.7$ & 0.78 & $10.8 \pm 0.1$ & $10.9 \pm 0.2$ & & 0.79 & $9.6 \pm 0.1$ & $9.4 \pm 0.2$ & $10.5 \pm 0.7$ & 0.59 \\
\hline Posterior wall, mm & $8.5 \pm 0.04$ & $8.5 \pm 0.1$ & $9.5 \pm 0.6$ & 0.57 & $9.0 \pm 0.1$ & $9.0 \pm 0.2$ & & 0.90 & $8.0 \pm 0.1$ & $8.1 \pm 0.1$ & $9.0 \pm 0.5$ & 0.45 \\
\hline LVEDD, mm & $47.9 \pm 0.1$ & $47.9 \pm 0.4$ & $45.9 \pm 2.0$ & 0.72 & $50.1 \pm 0.2$ & $49.9 \pm 0.6$ & & 0.70 & $45.8 \pm 0.2$ & $45.8 \pm 0.5$ & $43.6 \pm 1.9$ & 0.77 \\
\hline \multicolumn{13}{|l|}{ eGFR measurements $^{c}$} \\
\hline $\mathbf{N}$ & 954 & 115 & 5 & & 497 & 52 & 1 & & 457 & 63 & 4 & \\
\hline eGFR, $\left(\mathrm{ml} / \mathrm{min} / 1.73 \mathrm{~m}^{2}\right)$ & $108.2 \pm 0.7$ & $108.8 \pm 2.0$ & $119.5 \pm 9.5$ & 0.61 & $109.2 \pm 0.9$ & $114.5 \pm 2.8$ & $140.8 \pm 19.9$ & 0.045 & $107.2 \pm 1.1$ & $104.0 \pm 2.8$ & $112.7 \pm 11.3$ & 0.37 \\
\hline
\end{tabular}

Values are adjusted least square means \pm SE, except for age, BMI, and diabetes. ${ }^{\text {a}}$ Values are adjusted for age, BMI, gender (combined group). ${ }^{2 b}$ Values are adjusted for age, BMI, SBP, and gender (combined group). ${ }^{c}$ Values are adjusted for age, diabetes, and gender (combined group). Two-tailed descriptive p-values from t-test for independent data for the analysis using a dominant model, i.e. comparing CYP3A5*3/*3 individuals with CYP3A5*3/*1+CYP3A5*1/*1 individuals are given.

LVMI indicates left ventricular mass index; LVEDD, left ventricular end-diastolic diameter; eGFR, estimated glomerular filtration rate. 
Table 2. Baseline characteristics of the study population stratified by gender and hypertension status.

\begin{tabular}{lcccc}
\hline & \multicolumn{2}{c}{ Men } & \multicolumn{2}{c}{ Women } \\
\hline & Normotensive & Hypertensive & Normotensive & Hypertensive \\
\hline $\mathbf{N}$ & 335 & 219 & 388 & 142 \\
Age, years & $46.6 \pm 13.2$ & $52.9 \pm 13.6$ & $45.4 \pm 12.5$ & $56.9 \pm 10.1$ \\
BMI, $\mathrm{kg} / \mathrm{m}^{2}$ & $26.0 \pm 3.0$ & $27.8 \pm 3.5$ & $25.0 \pm 3.9$ & $27.7 \pm 4.8$ \\
SBP, $\mathrm{mm} \mathrm{Hg}$ & $124.4 \pm 9.3$ & $150.7 \pm 13.4$ & $118.7 \pm 11.0$ & $152.1 \pm 14.0$ \\
DBP, $\mathrm{mm} \mathrm{Hg}$ & $77.0 \pm 7.6$ & $90.5 \pm 9.8$ & $73.8 \pm 8.0$ & $88.7 \pm 10.6$ \\
Diabetes, $\mathrm{n} \mathrm{( \% )}$ & $8(2.4)$ & $9(4.1)$ & $6(1.5)$ & $3(2.1)$ \\
\hline
\end{tabular}

Data are mean values \pm standard deviation or number of individuals and percent.

SBP indicates systolic blood pressure; DBP, diastolic blood pressure; BMI, body mass index. 
Table 3. Genotype distribution of CYP3A5 genotypes, CYP $3 A 5 * 1$ allele frequency in men and women with and without arterial hypertension and OR $(95 \% \mathrm{CI})$ for hypertension.

\begin{tabular}{lcccc}
\hline & \multicolumn{2}{c}{ Men } & \multicolumn{2}{c}{ Women } \\
& Normotensive & Hypertensive & Normotensive & Hypertensive \\
\hline All individuals & 335 & 219 & 388 & 142 \\
Genotype & & & & \\
CYP3A5*3/*3, n (\%) & $304(90.7)$ & $197(90.0)$ & $335(86.3)$ & $128(90.1)$ \\
CYP3A5*3/*1, n (\%) & $31(9.3)$ & $21(9.6)$ & $50(12.9)$ & $13(9.2)$ \\
CYP3A5*1/*1, n (\%) & 0 & $1(0.4)$ & $3(0.8)$ & $1(0.7)$ \\
CYP3A5*1 allele frequency & 0.046 & 0.053 & 0.072 & 0.053 \\
OR $(95 \% \mathrm{CI}), \mathrm{p}$-value & $1.26(0.69-2.32), \mathrm{p}=0.46$ & $0.85(0.43-1.67), \mathrm{p}=0.63$ \\
Combined men and women & \multicolumn{5}{c}{$1.02(0.66-1.60), \mathrm{p}=0.92$} \\
OR $(95 \% \mathrm{CI}), \mathrm{p}$-value & \multicolumn{5}{c}{} \\
\end{tabular}

OR $(95 \% \mathrm{CI})$ for hypertension, adjusted for age, BMI, and gender were computed for the analysis comparing СYРЗА5*3/*3 (reference) individuals with CYP3A5*3/*1 + CYP3A5*1/*1 individuals. Two-tailed descriptive pvalues from WALD- $\chi^{2}$-test are given. 